\title{
A Novel Concept for Nano-Selenium Supplementation to Fight against COVID-19
}

\author{
Iqra Bano ${ }^{1, *}$, Abdul Rasheed Baloch², Safia Arbab ${ }^{3}$, Hira Sajjad ${ }^{4}$, Mir Sajjad Hussain Talpur ${ }^{5}$, Asma \\ Khalid $^{6}$ \\ ${ }^{1}$ Faculty of Bio-sciences, SBBUVAS, Sakrand, Sindh, Pakistan. \\ ${ }^{2}$ Laboratory of Fish Genetics, Institute of Animal Physiology and Genetics, Academy of Sciences of Czech Republic, Libechov, \\ Czech Republic. \\ ${ }^{3}$ Key Laboratory of Veterinary Pharmaceutical Development, Ministry of Agriculture, Chinese Academy of Agriculture Sciences, \\ Lanzhou 730050, China. \\ ${ }^{4}$ Faculty of Animal Husbandry \& Veterinary Sciences, Sindh Agriculture University, Tandojam, Pakistan. \\ ${ }^{5}$ Institute of Information Technology Center, Sindh Agriculture University, Tandojam, Pakistan. \\ ${ }^{6}$ District Headquarter Hospital, Mirpur Mathelo, Ghotki, Sindh, Pakistan.
}

\author{
How to cite this paper: Iqra Bano, Abdul \\ Rasheed Baloch, Hira Sajjad, Mir Sajjad \\ Hussain Talpur, Asma Khalid. (2021) A \\ Novel Concept for Nano-Selenium \\ Supplementation to Fight against \\ COVID-19. International Journal of \\ Clinical and Experimental Medicine \\ Research, 5(3), 264-268. \\ DOI: 10.26855/ijcemr.2021.07.006 \\ Received: April 13, 2021 \\ Accepted: May 8, 2021 \\ Published: May 28, 2021 \\ *Corresponding author: Iqra Bano, \\ Faculty of Bio-sciences, SBBUVAS, \\ Sakrand, Sindh, Pakistan. \\ Email: iqrashafi05@yahoo.com
}

\begin{abstract}
The 2019 pandemic of coronavirus (COVID-19) represents a serious threat to global health. Recently, the food practices in most developed countries have focused on the availability of basic nutrients in form of both macro and micronutrients in the diets to combat various diseases. The Selenium (Se) is a trace mineral known for its antioxidant and immunogenic abilities, one of the major trace elements in the human body that induces a strong antiviral effect. Nanotechnology has changed the perception of discovery and development of drugs in the recent three decades by opening up many hidden doors in pathophysiology and treatment options for diseases. The Nano-Se has gained considerable attention because of its unique antimicrobial activity. The current Se status in the fight against various viruses and the potential application of Nano-Se to combat COVID-19 are emphasized here in response to the global pandemic.
\end{abstract}

Keywords

Selenium, COVID-19, Nano-Se, Virus

\section{Introduction}

In Wuhan, province of Hubei, China, several cases of unknown respiratory infection have been found in hospitals since December 2019 with a history of exposure to seafood markets. Later on, scientists verified the syndrome as a severely acute coronavirus respiratory disease: coronavirus 2 (SARS-CoV-2) [1]. Todate, a major threat has been placed on human health around the globe by the subsequent 2019 coronavirus (COVID-19) Pandemic. Government agencies have frequently taken measures to prevent COVID-19 infection via self-isolation, social distancing, masking, and temperature monitoring. Researchers and health workers are putting all their efforts into developing the methods for diagnosis, treatment, and defense against COVID-19 by focusing on the discovery of antiviral drugs and vaccines [2]. Structurally the COVID-19 contains major three types of proteins which are: small envelope glycoprotein (E, Envelope Protein), spike (S, Spikes Protein) and membrane glycoprotein, and a few types of hemagglutinin esterase (HE protein). The gene of COVID-19 is a single-stranded RNA that survives more frequently than DNA viruses [3]. 
Selenium (Se) is one of the essential trace elements performing various processes of the body [4]. It is typically available as selenoproteins (SePs) approximately 3\% of which take part in glutathione peroxidase (GSH-Px) formation [5]. It performs various functions in the body; it maintains male and female reproductive physiology, acts as a regenerative agent, and fight against oxidative stress [6]. It can improve the body's immune function by enhancing the role of T cell (T cell) and cellular natural killer cells (NK cell) which destroy tumor cells [7]. Different functions of Se are elaborated in Figure 1. Nanotechnology has changed the perception of the discovery and development of drugs in the recent three decades by opening up many hidden doors in pathophysiology and treatment options for diseases [8]. The Nano-Selenium (Nano-Se) is a low-toxic and more bioactive form of Se which possess has high efficacy in the prevention of oxidative damage in comparison with other forms of Se [9]. According to previous studies, the Nano-Se can efficiently scavenge free radicals at less than $0.5 \mathrm{~mm}$ concentration. Currently, the Nano-Se is used in treating many conditions including liver fibrosis, various forms of cancer, viral infections, and checking the toxicity of drugs [10].

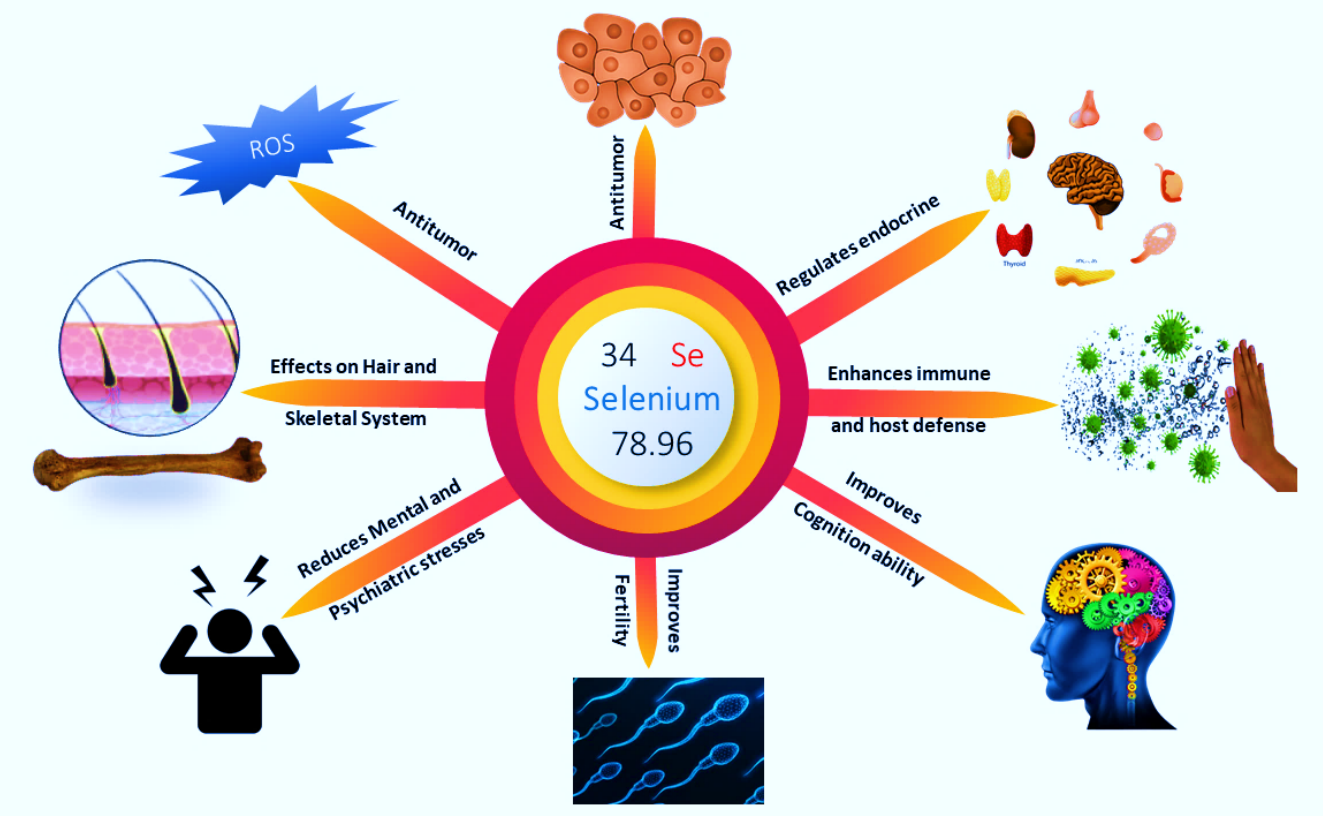

Figure 1. The health benefits and involvements of Se in various body systems.

\section{Effects of Se in various viral infections}

Se has long been directly involved in fighting against several viruses such as influenza, herpes simplex virus type 1 (HSV-1), hepatitis C virus (HCV), the coxsackievirus, and human immunodeficiency virus (HIV) [11]. Interestingly enough, beneficial effects of Se have been reported almost only on RNA virus infections, whereas Se and DNA virus information remains scarce [12]. Until now, various intervention investigations were conducted by using a single dose of Se or in combination with other trace minerals to observe its relevance with viruses and its therapeutic effects against viral infections (Table 1). The Se plays a significant role in immunity via reducing inflammation and oxidative stress [4]. Studies have shown that higher Se blood levels are associated with improved immune response [13]. Some investigations suggested that the mild intake of Se might raise the effects of TNF- $\alpha$ and interferon-gamma that may enhance the immune response against influenza viruses (H1N1). Therefore, it provides a basis to help prevent and treat viral illnesses, as it can enhance the immune system and has antioxidant properties ensuring the normal redox regulatory capacity to resist pathogen invasion [14]. The Se-food supplementation also increased the cytotoxicity of CD8+ T cells, by increasing the number of cells peripheral blood lymphocytes in human beings [15]. Some researchers suggest that sufficient Se can be the key to prevent virus-induced apoptosis from West Nile virus (WNV) infections [16]. The average Se content of cervical tissue was lower in patients infected with human papilloma infection (HPV) [17]. A dietary complement of humans at risk for modest Se deficiency could serve as an affordable and wide-ranging virus-infection adjuvant treatment in many countries in Europe and Africa and some regions of East Asia. Se deficiency causes defense destabilizing by reducing the expression of SePs. Severe interstitial pneumonitis was induced by Se deficiency among influenza-infected mice compared with Se-appropriate mice [11]. Besides, the lytic activity of human periphery blood lymphocytes and mouse 
spleen in NK cells was increased by Se supplementation [18]. The function of cytotoxic effector cells in COVID-19 can therefore be improved by Se supplementations [16].

Table 1. Various viral infections which are affected with Se intake

\begin{tabular}{cccc}
\hline Virus & Abbreviation & Study model & References \\
\hline West Nile Virus & WNV & Human & {$[16]$} \\
Hepatitis C Virus & HCV & Human & {$[23]$} \\
Herpes Simplex Virus & HSV-1 & Human & {$[24]$} \\
Human Papilloma Virus & HPV & Human & Human \\
Human Immunodeficient Virus & HIV & Chicken & {$[25]$} \\
Influenza Virus & HIN1 & Bovine & {$[26]$} \\
Respiratory Syncytial Virus & RSV & Human & {$[28]$} \\
Coxsackie Virus & CV &
\end{tabular}

\section{The Nano-Sesupplementation to fight against COVID-19}

Recently, small-scale nutritional status studies on COVID-19 patients have shown that in patients with and without pneumonia there is a significant lack of vitamin D and Se. According to some studies, it was revealed that Se deficiency is also associated with increased COVID-19 mortality, and a sufficient Se level is important for disease recovery [11]. Recently, few appropriate implications are being suggested by the European Society of Clinical Nutrition and Metabolism (ESPEN) for the management of COVID-19 patients. The recommendations were aimed to protect malnourishment by providing a sufficient quantity of macronutrients to fulfill the standards for lipids, protein, carbohydrates, and energy. Besides, the prevention of viral infection also requires adequate micro-nutrient supplementation. The low levels of micronutrients like vitamins B6, Se, and Zinc were associated with viral infections with adverse clinical results [8]. It was also found that COVID-19 is directly related to Se consumption. For example, in Enshi the cure rate for COVID-19 patients was found to be 36.4\% while in other Hubei towns the overall cure rate was $13.1 \%$. The Heilongjiang province, however, had a $2.4 \%$ mortality in North-Eastern China because of COVID-19. It was reported that Se levels were only $0.26 \mathrm{mg} / \mathrm{kg}$ in the hair of Heilongjiang Songnen Plain. These experimental observations suggest that cure rates are strongly correlated with the intakes of Se in cities outside of Hubei. The larger the Se consumption, the stronger the COVID-19 cure rate. Hence, the risk factor of COVID-19 mortality maybe because of Se deficiency [19]. In Germany, a cross-sectional study has shown that the serum level of Se in surviving COVID-19 patients is substantially higher than that in deceased COVID-19 patients [20]. Blood coagulation in patients with COVID-19 can increase mortality [21]. Increased tissue damage, infection and organ failure, and increased ratio of ICU deaths were associated with lower plasma Se concentration. In the plasma, Se level, the minimum platelet count, the minimum plasma anti-thrombin activity, and protein C activity were also positively correlated in COVID-19 patients [11].

The principal concern of using Se to combat various viruses is its high-dose poisonousness. Nano-Se was suggested to treat various diseases such as cancers and Huntington's disease. The persistence in the body of cytokine-induced killer (CIK) cells can also be effectively increased by Nano-Se. For example, more NK cells may be encouraged to infiltrate the tumor by combining Nano-Se and CIK cells, which activates a strong immune response for effective cancer immunotherapy [5]. Functional Nano-Se can also be developed for efficient use in the fight against SARS-CoV-2. The Ebselen, a species of organic Se, was revealed to inhibit COVID-19 via covalent binding to the virion through the plasma membrane. It is not very cytotoxic in humans and has proved safe and most effective at $10 \mu \mathrm{M}$ concentration with infected COVID-19 cells [22]. Nano-Se also is biocompatible, allowing it to an antiviral medicine's carrier [9]. It is therefore promising in the fight against COVID-19 to consider these merits of Nano-Se. However, very few experiments with Nano-Se against the COVID-19 are still being conducted. More studies are needed to confirm Se and in particular Nano-Se in patients with COVID-19.

\section{Conclusion and perspectives}

Se has been reported to combat various viruses for many years and has also been shown to correlate with positive results in patients with COVID-19. Ebselen has been confirmed to have an antiviral effect on SARS-CoV-2. Due to 
the low toxicity, antioxidant and immune-boosting ability, and other merits of Nano-Se, further research on the use of SARS-CoV-2 and improvements in the health consequences in COVID-19 patients is desirable and reasonable. We hope that, soon, Se and particularly Nano Se can play a vital role to combat COVID-19.

\section{References}

[1] H. Ge, et al. (2020). “The epidemiology and clinical information about COVID-19,” Eur. J. Clin. Microbiol. Infect. Dis., vol. 39, no. 6, pp. 1011-1019, 2020, doi: 10.1007/s10096-020-03874-z.

[2] S. Eslamkhah, N. Alizadeh, K. Hajiasgharzadeh, and A. Mokhtarzadeh. (2021). "The clinical characteristics and treatment approaches of COVID-19 : a concise review,” no. February, 2021, doi: 10.34172/PS.2021.6.

[3] S. Ghosh, E. Mostafavi, and A. Vernet-crua, "Molecular Nanotechnology Nanomaterial Approaches for the Prevention, Diagnosis and Treatment of COVID-19 : A Paradigm Shift," no. September, 2020, doi: 10.13140/RG.2.2.20501.76005.

[4] I. Bano, et al. (2019). "Effect of dietary selenium yeast supplementation on morphology and antioxidant status in testes of young goat,” Pak. J. Zool., vol. 51, no. 3, 2019, doi: 10.17582/journal.pjz/2019.51.3.979.988.

[5] C. Liu, H. Lai, and T. Chen. (2020). "Boosting Natural Killer Cell-Based Cancer Immunotherapy with Selenocystine/Transforming Growth Factor-Beta Inhibitor-Encapsulated Nanoemulsion,” ACS Nano, vol. 14, no. 9, pp. 11067-11082, 2020, doi: 10.1021/acsnano.9b10103.

[6] I. Bano, et al. (2018). "Effect of Dietary Selenium Supplementation on Morphology and Antioxidant Status in Testes of Goat," J. Basic Appl. Sci., vol. 14, no. March, pp. 53-61, 2018, doi: 10.6000/1927-5129.2018.14.08.

[7] S. Hariharan and S. Dharmaraj. (2020). "Selenium and selenoproteins: it’s role in regulation of inflammation,” Inflammopharmacology, vol. 28, no. 3, pp. 667-695, 2020, doi: 10.1007/s10787-020-00690-x.

[8] C. Weiss, et al. (2020). "Toward Nanotechnology-Enabled Approaches against the COVID-19 Pandemic,” ACS Nano, vol. 14, no. 6, pp. 6383-6406, 2020, doi: 10.1021/acsnano.0c03697.

[9] A. M. Sheiha, et al. (2020). "Effects of dietary biological or chemical-synthesized nano-selenium supplementation on growing rabbits exposed to thermal stress,” Animals, vol. 10, no. 3, pp. 1-16, 2020, doi: 10.3390/ani10030430.

[10] S. Menon and V. K. Shanmugam. (2020). "Chemopreventive mechanism of action by oxidative stress and toxicity induced surface decorated selenium nanoparticles,” J. Trace Elem. Med. Biol., vol. 62, p. 126549, 2020, doi: 10.1016/j.jtemb.2020.126549.

[11] L. He, et al. (2021). “Using nano-selenium to combat Coronavirus Disease 2019 (COVID-19)?,” Nano Today, vol. 36, p. 101037, 2021, doi: 10.1016/j.nantod.2020.101037.

[12] E. W. Taylor. (2020). "RNA Viruses vs. DNA Synthesis: A General Viral Strategy That May Contribute to the Protective Antiviral Effects of Selenium,” Preprints, no. June, p. n/a, 2020, doi: 10.20944/preprints202006.0069.v1.

[13] M. Iddir, et al. (2020). "Strengthening the immune system and reducing inflammation and oxidative stress through diet and nutrition: Considerations during the covid-19 crisis,” Nutrients, vol. 12, no. 6, pp. 1-39, 2020, doi: 10.3390/nu12061562.

[14] M. Bae and H. Kim. (2020). "Mini-Review on the Roles of Vitamin C, Vitamin D, and Selenium in the Immune System against COVID-19,” Molecules, vol. 25, no. 22, pp. 1-12, 2020, doi: 10.3390/molecules25225346.

[15] B. Muszyńska, et al. (2020). "Preliminary study on Se-enriched Lentinula edodes mycelium as a proposal of new feed additive in selenium deficiency,” PLoS One, vol. 15, no. 5, pp. 1-19, 2020, doi: 10.1371/journal.pone.0233456.

[16] G. Bermano, C. Méplan, D. K. Mercer, and J. E. Hesketh. (2020). "Selenium and viral infection: Are there lessons for COVID-19?,” Br. J. Nutr., pp. 618-627, 2020, doi: 10.1017/S0007114520003128.

[17] C. De Luca, Z. Kharaeva, D. Raskovic, P. Pastore, A. Luci, and L. Korkina. (2012). “Coenzyme Q 10, vitamin E, selenium, and methionine in the treatment of chronic recurrent viral mucocutaneous infections,” Nutrition, vol. 28, no. 5, pp. 509-514, 2012, doi: 10.1016/j.nut.2011.08.003.

[18] S. Y. Wu, T. Fu, Y. Z. Jiang, and Z. M. Shao. (2020). “Natural killer cells in cancer biology and therapy,” Mol. Cancer, vol. 19, no. 1, pp. 1-26, 2020, doi: 10.1186/s12943-020-01238-X.

[19] D. Editor, et al. (2020). “Letters to the Editor,” pp. 1297-1299, 2020.

[20] M. Majeed, K. Nagabhushanam, S. Gowda, and L. Mundkur. (2021). "An exploratory study of selenium status in healthy individuals and in patients with COVID-19 in a south Indian population: The case for adequate selenium status,” Nutrition, vol. 82, p. 111053, 2021, doi: 10.1016/j.nut.2020.111053.

[21] P. Bacteria. “Open Life Sciences Bactericidal and Antioxidant Activity of Saudi Domestic Essential Oils on Certain Pathogenic Bacteria Cover Letter of Reviewers’ comments Dear Editor and Reviewer 4 Open Life Sciences: Thank you for accepting the manuscript article”.

[22] H. Sies and M. J. Parnham. (2020). "Potential therapeutic use of ebselen for COVID-19 and other respiratory viral infections,” Free Radic. Biol. Med., vol. 156, pp. 107-112, 2020, doi: 10.1016/j.freeradbiomed.2020.06.032. 
[23] S. Gül-Klein, et al. (2021). "Serum Selenium Status as a Diagnostic Marker for the Prognosis of Liver Transplantation,” Nutrients, vol. 13, no. 2, p. 619, 2021, doi: 10.3390/nu13020619.

[24] C. Wang, et al. (2020). "The Inhibition of H1N1 influenza virus-induced apoptosis by functionalized Selenium nanoparticles with $\beta$-Thujaplicin through ROS-mediated P53 and AKT signaling pathways,” 2020, doi: 10.21203/rs.3.rs-15986/v1.

[25] I. A. Osuna-Padilla, et al. (2020). "Zinc and selenium indicators and their relation to immunologic and metabolic parameters in male patients with human immunodeficiency virus,” Nutrition, vol. 70, 2020, doi: 10.1016/j.nut.2019.110585.

[26] B. Shojadoost, et al. (2020). "Supplemental dietary selenium enhances immune responses conferred by a vaccine against low pathogenicity avian influenza virus,” Vet. Immunol. Immunopathol., vol. 227, no. June, p. 110089, 2020, doi: 10.1016/j.vetimm.2020.110089.

[27] Maiti and Bidinger. (1981). “No Title No Title,” J. Chem. Inf. Model., vol. 53, no. 9, pp. 1689-1699, 1981.

[28] L. Hiffler and B. Rakotoambinina. (2020). "Selenium and RNA Virus Interactions: Potential Implications for SARS-CoV-2 Infection (COVID-19),” Front. Nutr., vol. 7, no. September, pp. 1-10, 2020, doi: 10.3389/fnut.2020.00164. 